\title{
Suggestion of Design Evaluation Plan based on Star Life Cycle to introduce the Information Minimalism Concept of KOREA Nuclear Plant
}

\author{
Gwi-sook Jang a*, Seung-min Lee ${ }^{\mathrm{b}}$, Gee-yong Park ${ }^{\mathrm{a}}$ \\ ${ }^{a}$ I\&C and HFE Research Division, Korea Atomic Energy Research Institute, 989-111, \\ Daedeok-daero, Yuseong-gu, Daejeon 305-353, Republic of Korea \\ ${ }^{b}$ Maritime Reactor Development Center, Korea Atomic Energy Research Institute, 989-111, \\ Daedeok-daero, Yuseong-gu, Daejeon 305-353, Republic of Korea
}

\begin{abstract}
The design of Korea Nuclear Power Plant (NPP) main control rooms (MCR) has been changed to be fully digitalized. Five or six display devices are assigned to each operator in NPP MCR to provide the information of safety parameter and plant status, and various control functions by connecting computerized control devices. Under this circumstance, the distributed displays can induce a dispersion of the operators' attention and increase the workload while conducting monitoring and control tasks efficiently. In addition, to support human operators to reduce their workload and increase the performance, the concepts of the ecological interface design (EID) and the operator-centered design were applied to the design HMI display. However these designs are applied to a limited set of screens and did not differ largely from the traditional HMI design in that the layout of the information is somewhere similar to P\&IDs.

In this paper, we propose a design evaluation plan based on star life cycle to introduce the information minimalism concept for designing an HMI display.
\end{abstract}

Index Terms-Monitoring System, Human Factors Engineering, Human-Machine Interface

\section{INTRODUCTION}

$\mathrm{T}$ he traditional HMI (Human Machine Interface) design approach for a Korea NPP (Nuclear Power Plant) is a heavily mimic-based hard-paneled interface based on P\&ID (Piping and Instrumentation Diagram). Recently, the design of the Korea Nuclear Power Plant (NPP) main control rooms (MCR) was changed to be fully digitalized, as shown in Fig.1. Five or six display devices are assigned to each operator in an NPP MCR to provide the information of safety parameter and plant status, and various control functions by connecting computerized control devices.

This was expected to minimize the operator's movement and enable the operator to operate even when seated, greatly enhancing the operator's macro performance. On the other hand, because the information based on the existing P\&IDs was implemented as a hierarchical structure on the VDU (Visual Display Unit) with the display space constraint, it required additional tasks such as information navigation in addition to primary tasks such as monitoring, situation assessment, planning, and implementation.

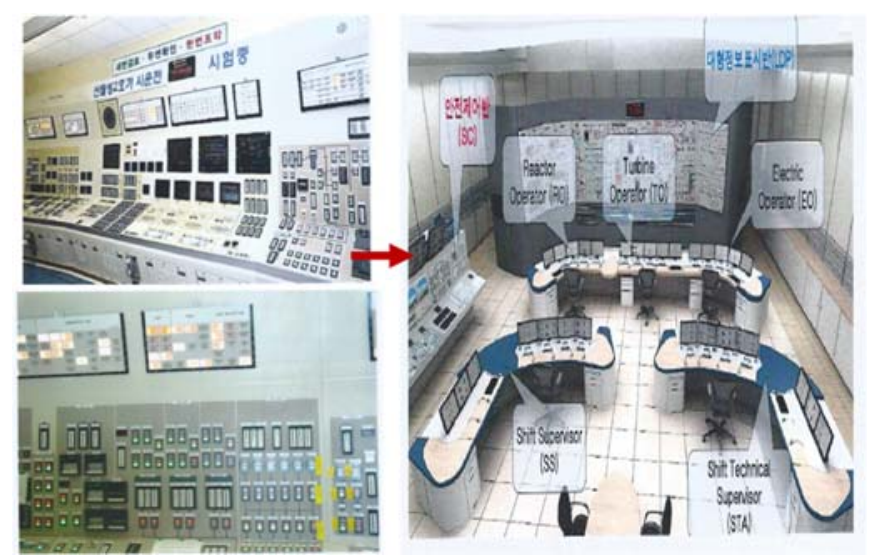

Fig. 1. Status of a main control room in Korean NPP

According to NUREG/CR-6633, as the interface is distributed over many displays limited in size, operators will have to navigate through them to access the information they are looking for. The display shown on each screen is chosen by the operator, e.g., a mimic-based display, trends, and alarm systems. While this flexibility offers some advantages, studies have shown that operators often get lost, experiencing a hard time managing screens and finding the information they are particularly looking for and thus reducing the operator performance $[1,2]$.

In practice, the information display of the digital based control room of a Korean NPP is operated with a hierarchy structure of system-oriented, component-oriented, and function-oriented based on existing P\&IDs. The operator obtains the monitoring and controller information necessary for operation through the information navigation by hierarchically distributed information over five or more display pages to perform a particular operation mode. As a result of the 
intervention of these additional tasks, the reaction time is delayed, and it is difficult to maintain the cognitive context, and can cause a cognitive error under a situation such as an emergency.

Therefore, it is necessary that an information structure and visualization shall be designed to minimize the cognitive burden of recognition, memory, and judgment on the relationship between complex systems when an unexpected event occurs. The information structure and visualization design is based on a careful analysis and understanding of the work domain of the operator.

P\&ID based layouts tend to require more display space. Overuse of mimic layouts can result in visual clutter. P\&ID based layouts of controls are usually less easily operated configurations than other array conventions. Thus, a display method that minimizes the P\&ID based display is emerging.

Although they are from different domains (e.g. process controls and nuclear power generation controls, etc.), common topics are raised issues and/or concerns of an HMI for plant operators. These common issues are summarized and translated as the following design aspects [3]:

- Minimalism: To support operator understanding and situation awareness, utilizing graphical representation of plant assets can be beneficial. However, unnecessary details of the graphics shall be avoided, and reduced to visual noise and clutter.

- Color palette: A color palette has been defined for the HMI to reduce operator's cognitive workload and make abnormal conditions more apparent.

- Visual coding: A visual coding refers to any visual treatments to indicate or imply certain context around data.

- Screen hierarchy: Categorizing the screens systematically can improve the navigation efficiency, and, moreover, describes more details of the process and/or assets.

- Navigation: Having multiple forms of navigation improves the operator's speed of screen transition and awareness of screen locations.

- Information layout: As operators form their mental model of the operation (processes and assets) based on the location of the physical equipment and assets in the plants, utilizing the P\&ID for process overview to a certain extent can be useful.

- Contextual data: Data can transform into useful information when provided with a proper context, representing the context of the data, (e.g., targets, operating ranges, alarm limits, process relationship) supports faster operator understanding and situation awareness.

The above concept of an HMI design was estabilshed as shown in Fig.2.

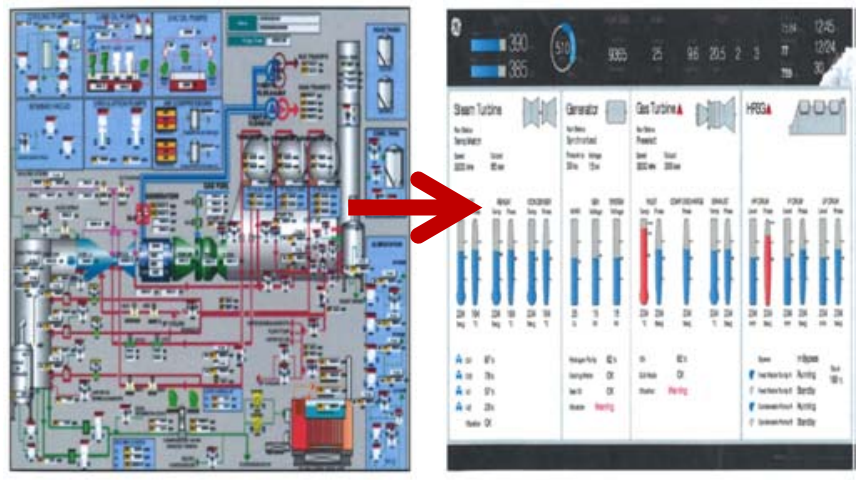

Fig.2. An example of resulting plant overview screen

However, the HMI design that minimize the P\&ID as based on the above example has not yet proven its effectiveness.

To solve the above problem, the designer has to decide how to reduce the amount of information to be displayed on the screen so that the user can work easily. In addition, it is important to provide a means for the operator to easily move the information display space and naturally make a cognitive transition in the display design with a large amount of information networks.

Thus, we propose a design evaluation plan based on the star life cycle to introduce the information minimalism concept for designing an HMI display. These days a minimalism concept and the star life cycle methodology is emerging in the HMI design approach.

Minimalism is used to support the operator understanding and situation awareness, and utilizing a graphics representation of plant assets can be beneficial. However, unnecessary details of the graphics shall be avoided to reduce visual noise and clutter.

This paper proposes a monitoring and controller display method for each operation mode based on log analyses of operator actions as an implementation method of minimalism. This method minimizes the existing information navigation by providing the monitoring and control means necessary for the operator to perform a specific operation mode on one or two display pages.

The method provides the monitoring and controller information for each operation mode of NPP to operator based on the understanding of the work domain of the operator. And the method complements the monitoring information and the control means for each operation mode through analysis of the operation behavior log obtained during operation to improve the efficiency of the display for each operation mode.

The star life cycle methodology, suggested by Hartson and Hix, is an evaluation-centered iterative usability engineering process. The star lifecycle does not specify any ordering of 
activities. In fact, the activities are highly interconnected: you can move from any activity to any other, provided you first go through the evaluation activity. This reflects the findings of the empirical studies.

An evaluation is central to this model, and where an activity is completed, its results must be evaluated. It is expected that the suggested evaluation plan will be valid to estimate an applicability of various HMI design-related theories, and the HMI design-related theoretical features evaluated by the suggested evaluation will be reflected in the new monitoring and control system design.

\section{INFORMATION MINIMALISM CONCEPT}

Fig. 3 shows the monitoring and controller display method for each operation mode based on $\log$ analyses of the operator actions, and the detailed descriptions of each module are as follows.

\section{A. Monitoring and controller DB for each plant mode}

The monitoring and controller database for each operation mode is composed of the initial analysis information and complementary information based on the operation log analysis during operation. The initial analysis information means the basic information of the monitoring and the controller to be provided to the operator for each operation mode.

This was confirmed through the task analysis of the operator for an NPP. The complementary information is the condition evaluation and the action data for each the operation mode based on the operation behavior log analysis.

In addition, the initial analysis information and complementary information is stored in the database in the form of ECA rules (on Event if Conditions then Action). Basic display information for each operation mode and complementary information based on operation behavior analysis are added / deleted / modified to the shared memory through the rule interface, which is an operator interface module.

The rules interface is an interface for system users and designers to define, manage, delete, and modify the display rules.

\section{B. Monitoring and controller management module for each operation mode}

The management module is a main module that drives the monitoring and controller database to provide the information indicator with the monitoring and the control information necessary for the task in each operation mode. This module consists of a display condition evaluator for each operation mode and a display actor for each operation mode.

If the operator selects a specific operation mode, the management module provides necessary information for the corresponding monitoring and controller means to the operator. In addition, this module is responsible for running the operator's demands.

The display condition evaluator for each operation mode is connected to the event and rule triggering to sequentially drive the display rule. Then, the condition of this rule is evaluated, and if the condition is satisfied, the action of the rule is executed.

\section{Operation behavior log analysis and complementary module}

To complement the monitoring and control information required by the operator according to the operation mode, this module logs and stores the object and control means monitored by the operator according to the operation mode. Then this module preprocesses and performs statistics and correlation analysis on periodically stored log information.

The analysis result is prepared based on the condition evaluation and action data for displaying necessary information for each operation mode. The complementary module generates only the data to be supplemented during online operation and reflects the complementary data to the system after confirming the operator according to the administrative procedure.

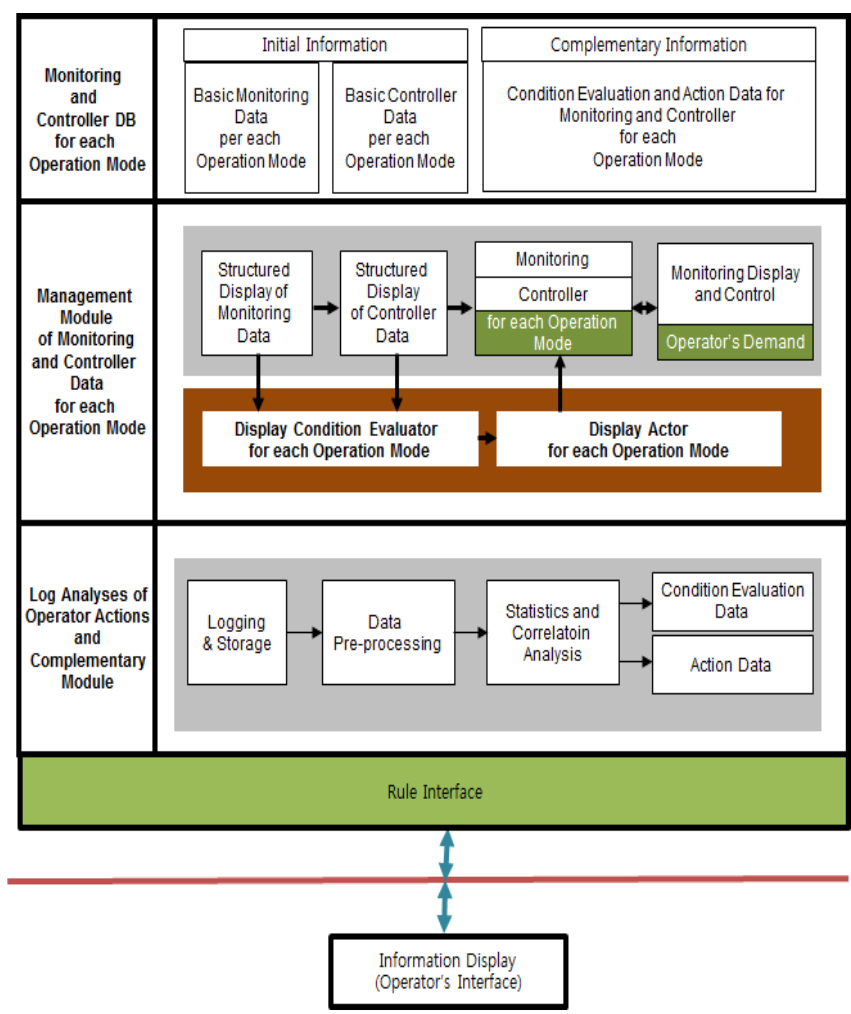

Fig. 3. A Monitoring and Controller Display Method for each Operation Mode based on Log Analyses of Operator Actions [5]

\section{OPERATION SEQUENCE}

1) As shown in Fig. 3, the "monitoring and controller DB for 
each operation mode" is a database composed of ECA rules. The monitoring and controller information to be provided to the operator for each operation mode are confirmed by the operator's task analysis at the beginning of the monitoring system design are stored in advance as ECA (on event if condition then action) rules.

2) When the operator selects the start operation mode button after the monitoring system starts to operate, the monitoring system activates the monitoring information and controller manager module for each operating mode based on the "initial analysis information ECA DB". The monitoring information and control means necessary for performing the specific driving task are provided in a screen of 1 to 2 pages. In particular, the management module comprises a condition evaluator for determining the display condition for each selected operation mode, and an activity unit for activating the display method for each operation mode that should be provided to the information display when the condition is satisfied.

3) In addition, all operations of the operator are logged and stored until the operator completes the related tasks after selecting the specific operation mode start button.

4) If operator select the specific operation mode completion button, logging and saving of the operator's action are stopped.

5) When the operation history of each operation mode is accumulated enough to analyze the statistical correlation at the time of the periodic test of the monitoring system, if the operator selects the log analysis button, the preprocessing for analyzing the stored $\log$ is performed and the following analysis is performed. And the result is provided to an information display.

- The number and frequency of monitoring and controllers are detected by the operator through screen navigation in addition to the screen provided previously for each specific operation mode

- Correlation between specific operating modes and additional monitoring and control periods with high frequency

6) Based on the results of the log analysis of the operator's action, the supplementary items (display condition and activity information for each operation mode) of the ECA DB are determined through surveys of the operators.

7) If the operation log analysis result is determined, the condition evaluation and activity information for each operation mode determined by the tool provided by the rule interface are input to the "ECA DB based on log analysis" according to the administrative procedure.

\section{Design Evaluation Plan Based on Star Life Cylce}

Making the user interface design and development process iterative is a way to ensure that users can get involved in the design, and that different kinds of knowledge and expertise related to the computer system can be brought into play as needed. This paper shall use an approach adapted from a model proposed by Hix and Hartson (1993). This is known as the star life cycle for obvious reasons, as you can see from its appearance in Fig 4.

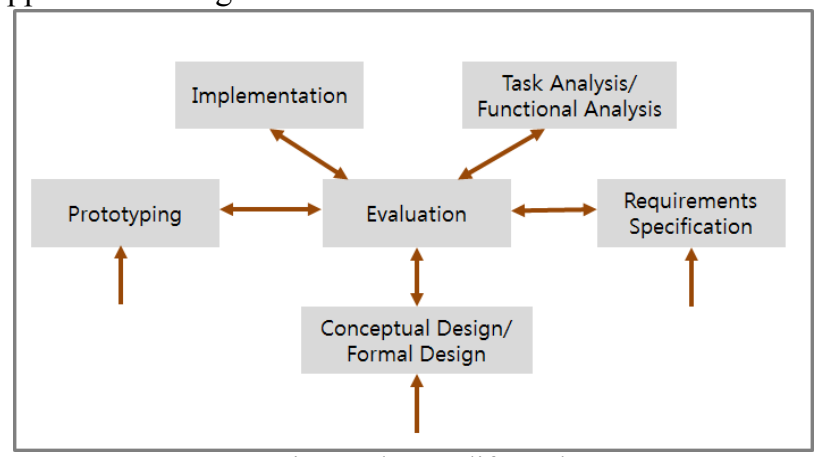

Fig. 4. The star life cycle

The central point of the star is an evaluation, which is viewed as being relevant at all stages in the life cycle and not just at the end of the product development as the classic life cycle tends to suggest. The evaluation is concerned with gathering data about the usability of a design or product by a specified group of users for a particular activity within a specified environment or work context. Users should be involved in every part of the user interface design and development life cycle [4].

- Early in the design process when the requirements are being specified

- During prototyping, to test designs and options

- During training/after delivery of the system

This paper proposes design evaluation plan based on stat life cycle. Monitoring system design for NPP consists of obtaining the design input data, setting the driving concept, developing a functional requirement, concept design, detailed design, testing, and evaluation (See Fig.5).

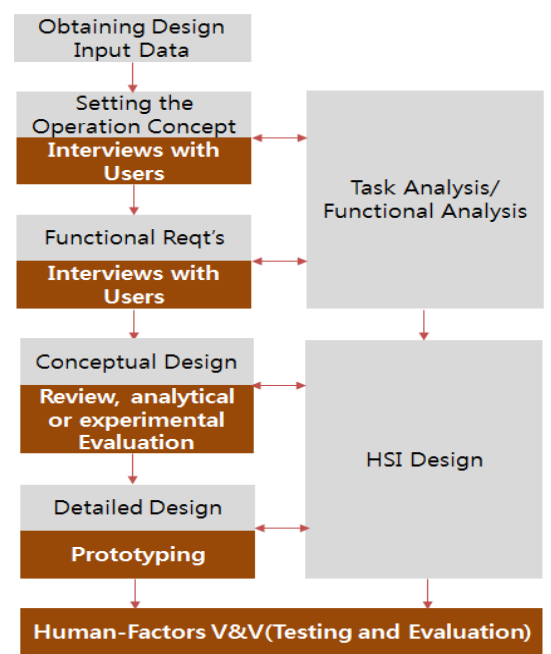

Fig. 5. Design steps based on star life cycle for monitoring system 
The monitoring system design work is carried out and integrated by the human-factors design team through the concept, basic and detailed design stages by receiving the results of the human-factors planning and analysis tasks. The design procedure and method of the nuclear plant monitoring system are as follows.

\section{A. Obtaining design input data}

Obtain the following input data for monitoring system design.

- Operator job requirements

- System requirements

- Regulatory requirements

\section{B. Setting the operation concept}

Set up the driving concept according to the structure and role of the nuclear plant operation group. The concept of driving includes the following contents.

- Verify the relationship between operator and plant automation

- Provide high-level description for operators to perform their duties using surveillance systems

- Mentioning of mutual relations among operators

\section{Development of function requirement for monitoring system}

The functional requirements of the monitoring system are developed to represent the operation concept, operator functions and duties, and operator requirements for safety. It also includes various kinds of detailed functional requirements for monitoring system such as alarm, indication, and control.

\section{Conceptual design of surveillance system}

The monitoring system performs a concept design according to the pre-established functional requirements for monitoring system. To meet the design requirements in this process, various design plans are examined considering the following items.

- Latest monitoring implementation technology

- Technological feasibility

- Human performance aspect

- Tradeoffs between monitoring technologies

The conceptual designs examined are selected through operational experience and a literature review, analytical or an experimental evaluation. In addition, the monitoring system performance requirements are identified for the selected conceptual designs.

\section{E. Detailed design of monitoring system}

In the detailed design stage of the monitoring system, detailed design requirements (hereinafter referred to as 'style guides') for specific monitoring systems are developed and applied to the features, layout and environmental design of the monitoring system screen and control systematically. The style guide is divided into high-level general guidelines for monitoring the system design and detailed design guidelines that apply specifically to specific plants. Detailed design guidelines that apply specifically to specific plants are developed to meet the baseline level of general guidelines. The design criteria for the detailed design of the monitoring system are based on the contents of the plant concept design, the standard used in the field of nuclear power plants, the industry standard or guidelines, and the general characteristics and practices of the monitoring system required by the plant operator.

The design criteria for the main detailed monitoring system design are as follows.

- Designed to support primary task requirements, such as plant monitoring and control, while minimizing the secondary workload required to operate monitoring system facilities

- Minimizes the possibility of human error and maximizes the detection capability

- Consider communication between operators, collaboration and workload, information feedback, on-site environment, inspection, test and maintenance task, and safety importance.

- Determine the monitoring screen layout considering roles of individual operators and various deployment strategies

- Designed to support the operator's task performance in all operation and operating environment conditions

- Designed to take into account situations in which the operator's ability to perform tasks may be degraded.

- Be able to support the work of inspection, maintenance, and testing, and to ensure that these tasks do not interfere with plant monitoring and control

\section{F. Testing and evaluation for monitoring system}

Testing and evaluation of monitoring system design is repeated throughout the entire development process of the monitoring system design. The test and evaluation are repeated in the human-factors verification and verification process, and the method is as follows.

- Trade-off evaluation: derive discrepancies through a styleguide-based, checklist-based evaluation and design alternatives to discrepancies. Analyze conflicts between initial design (plan) and alternative design (plan), and confirm design (plan) considering safety and humanfactors efficiency. If there are trade-offs in terms of operator performance for several designs, they are determined by the Analytic Hierarchy Process (AHP) 
method. The AHP method is applied to a large number of decision-making fields because it is convenient to convert the complex criteria into a simplified hierarchical structure and compares them to each other to rank the comparison objects. Therefore, the criteria for evaluation and 2 to 3 candidates for the design are ranked as follows in each design through each step of AHP, and objective results are obtained.

$\checkmark$ Operator's task requirements

$\checkmark \quad$ Operator performance and restrictions

$\checkmark \quad$ HSI performance requirements

$\checkmark \quad$ Inspection and testing requirements

$\checkmark$ Maintenance requirements

- Performance-based testing: A test that evaluates the performance of the operator after simulating the monitoring system design (draft). The monitoring system is evaluated in accordance with the conformance standards of the performance as follows.

$\checkmark \quad$ Purpose of test: Evaluate the feasibility of the monitoring system design (draft) according to the conformity criterion of the degree of performance as a method to evaluate the performance of the operator after simulating the design of a supervisory system.

$\checkmark \quad$ General method of test: The design evaluation of the monitoring system first defines the scope of the test environment, development schedule, development restrictions, and development related tests. This test defines verification test items and test limitations, selection and training of testee, selection of test scenarios, defining test scales and measurement methods, conducting preliminary tests, collection and analysis of test data, and analysis of the test results.

$\checkmark$ Requirements for test equipment: The requirements for the test equipment describe the structure of the test equipment, the functions of the equipment, the development and operation plans of the test equipment, and the requirements for the test plan. They also describe the hardware and software requirements of the test equipment, the development environment requirements, and the associated requirements and development limitations of developing the test equipment.

$\checkmark \quad$ Selection of performance evaluation method: the performance of the monitoring system design is evaluated by the accuracy and appropriateness of the information delivery time (operator response time) and the information transmitted to the operator. In addition, the acceptance criterion for performance evaluation is that the improved monitoring system design is 'allowed' if the supportability of the power plant situation to the improved supervisory system design is better than the level of task supportability in the interface environment of the existing main control room, and is evaluated as worthy. As the measurement variables of the performance evaluation test, the recognition time, recognition accuracy, objective situation recognition using SACRI (Situation Awareness Control Room Inventory), and subjective situation recognition using SART (Situational Awareness Rating Technique) are utilized.

$\checkmark \quad$ Selection and training of testee: Construct testee group using experts who have experience of operating a NPP. Experts belonging to the humanfactors design team or those with a high degree of experience or proficiency are excluded in order to reduce the bias of the tester, which may affect the test results. It produces training materials on the plant design and operation developed by experts such as the fluid, core, main machine and safety analysis field in the ergonomics design team. Prior to securing a plant evaluation test room, classroom education was conducted and practical training was conducted when the evaluation test was completed.

$\checkmark \quad$ The test design and data analysis methodology: The performance evaluation is based on a randomized factorial design. Verification of differences in the perceived performance time, perceptual accuracy, and SART Rating Scales was performed using the Paired Samples t-test. In addition, the verification of the difference of situation recognition through SACRI is verified by comparing the sensitivity ( $\mathrm{d}$ ') and the criterion (response criterion $(\beta)$ ) of the operator under test conditions $\mathrm{A}$ and $\mathrm{B}$. The sensitivity and judgment criteria are derived based on the SDT (Signal Detection Theory), and the statistical significance is obtained through normalization of the data collected through a SACRI evaluation.

\section{RESULTS AND FUTURE PLANS}

A traditional HMI design approach of an NPP was heavily based on the P\&IDs. A clear trend in Korea NPPs is to modernize the control rooms, moving from panel-based traditional control rooms to computerized solutions. A significant amount of work was done on the process control HMI design using an ecological interface design and the operator centered interface. These designs are applied to a limited set of screens and did not differ largely from the traditional HMI design in that the layout the information is somewhere similar to the P\&IDs.

The digital-based nuclear plant monitoring system has a problem in that there is a large amount of information to be 
displayed in a limited display space and that the capacity of the operator to process the visual information is limited. In order to solve the above problems, the latest digital technology and display technology have been introduced.

In this paper, we proposed a design evaluation plan based on star life cycle to introduce the information minimalism concept for designing HMI display. The information minimalism concept is the monitoring and controller display method for each operation mode based on log analyses of operator actions.

This method provides the monitoring information and control means necessary for the operator according to operation mode to perform a specific operation. This method can reduce the information searching process time and the transition between the display pages. So, this method can be provided as an alternative to overcome the problems of P\&IDs based hierarchical information provision.

This method will be validated by establishing a validation facility in the future. In addition, this concept is added to the concept of operator support with the existing display configuration and navigation as it is, and it extends its application range while actually utilizing it.

\section{REFERENCES}

[1] O'Hara, J., \& Brown, W. (2002), "The effects of interface management tasks on crew performance and safety in complex, computer-based systems", NUREG/CR-6633. Washington, D.C.:U.S. NRC.

[2] A.O.Braseth, C. Nihlwing, Lessons Learned from Halden Project Research on Human System, Nuclear Engineering and Technology, Vol.41 No.3, April, 2009.

[3] S.Y Kim, J.V.Wormer, Human-Machine Interface Design for Power Generation Plant Operators

[4] D.Stone, C.Jarrett, "User Interface Design and Evaluation", Morgan Kaufmann, 2005, pp. 19-20.

[5] Gwi-sook Jang, Seung-min Lee, Gee-yong Park, “A Monitoring and Controller Display Method for each Operation Mode based on Log Analyses of Operator Actions", Transactions of the Korean Nuclear Society Spring Meeting Jeju, Korea, May 18-19, 2017

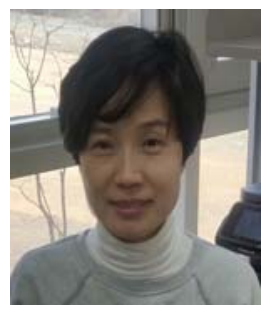

Gwi-sook. Jang was born in Daegu, Republic of Korea in 1967. She received her M.S. degree in computer science from the Kyungpook National University, Daegue, Korea in 1992. She received her $\mathrm{Ph} . \mathrm{D}$. in computer science from the Chungnam National University, Daejeon, Korea in 2010. She has worked for the Korea Atomic Energy Research Institute since February 1992. She has designed the monitoring system for a Small Modular Reactor (SMR) and a research reactor as principal researcher. Dr. Jang is a member of the Korean Nuclear Society

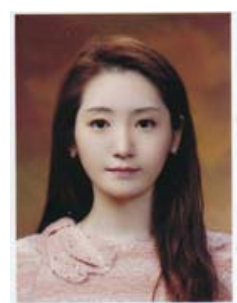

Seung M. Lee was born in Seoul, Republic of Korea in 1984. She received a B.S. degree in physics from Chung-Ang University, Seoul, in 2008 and an M.S. degree and Ph.D. in nuclear engineering from the Korea Advanced Institute of Science and Technology (KAIST), Daejeon, in 2010 and 2015. From 2015 to 2016, she was a Postdoctoral Researcher with the Mechanical Technique Laboratory in at KAIST. Since 2016, she has been a Senior Researcher with the Maritime Reactor Development Division, Korea Atomic Energy Research Institute (KAERI). Her research interests include autonomous operating system, information processing and cognitive load degradation for operators in nuclear power plants, and design of a Small Modular Reactor (SMR) control room and monitoring display.

Dr. Lee was a recipient of the International Symposium on Symbiotic Nuclear Powers System for the $21^{\text {st }}$ Century (ISSNP2013) Best Student Award in 2013.

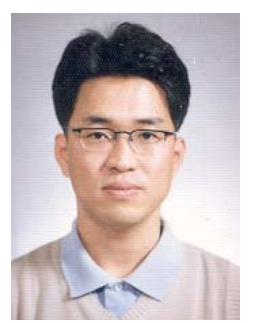

Gee-Yong Park received a B.S. degree in nuclear engineering from Hanyang University, Seoul, Korea, in 1990 and an M.S. degree and Ph.D in nuclear and quantum engineering from the Korea Advanced Institute of Science and Technology (KAIST), Daejeon, Korea, in 1992 and 1996, respectively. From 1996, he has been being involved at the Korea Atomic Energy Research Institute (KAERI) and is currently developing of instrumentation and control (I\&C) systems. His research interest includes the development of diagnosis and control methodologies for nuclear power plants, safety analysis of safety-critical software, and reliability analysis of digital systems. Dr. Park is a member of the Korean Nuclear Society. 